\title{
POSSÍVEIS EVIDÊNCIAS DA VITAMINA B12 NA DOR NEUROPÁTICA Seria um fator de proteção em Doenças Neurológicas?
}

\author{
Adalgiza Mafra Moreno \\ Doutorado em Ciências Cardiovasculares pela Universidade Federal Fluminense, Brasil \\ Coordenadora de Pesquisa da Universidade Iguaçu (UNIG), Brasil \\ adalgizamoreno1@gmail.com \\ Marco Orsini \\ Departamento de Neurologia, Universidade Iguaçu (UNIG), Brasil. \\ bentoorsini2013@gmail.com \\ Jacqueline Stephanie Fernandes do Nascimento \\ Graduanda de Medicina na Universidade Iguaçu (UNIG), Brasil. \\ jac.fn@hotmail.com \\ Nicolle dos Santos Moraes Nunes \\ Graduanda de Medicina na Universidade Iguaçu (UNIG), Brasil. \\ nicollemnunes18@gmail.com
}

\begin{abstract}
RESUMO
Introdução: A prevalência de pacientes acometidos por dor neuropática na população em geral pode chegar a $10 \%$. Quando comparado com demais síndromes de dor crônica, os pacientes com dor neuropática descrevem pior estado de saúde mental e física, tornando-se desafiador seu controle. Dado o visível quantitativo de doenças associadas à dor neuropática e as respostas restritas aos manejos atuais, revela-se a busca por novas terapêuticas a serem utilizadas como monoterapia ou em combinação com os tratamentos vigentes. Uma dessas terapêuticas baseia-se na suplementação com vitamina B12, ainda que não seja evidenciado sinais sugestivos de deficiência. Metodologia: Trata-se de estudo exploratório descritivo que emprega o método de revisão integrativa, categoria que consiste em explorar o conhecimento já definido, incluindo pesquisas com diferentes técnicas. Realizou-se em abril de 2020 o levantamento de artigos indexados nas bases de dados eletrônicas PubMed, MedLine, LILACS e BIREM. Resultados e Discussão: Há vários anos, a vitamina B12 tem sido empregada sob forma de monoterapia ou combinadas a demais fármacos para o tratamento de dor em diversas condições clínicas, como dor inflamatória, neuropática, doenças neurodegenerativas, radiculopatia, e etc. demonstrando evidências positivas. Conclusão: Dada as evidências, a vitamina B12 apresenta-se como um candidato potencial para o tratamento da neuropatia periférica com boa segurança e baixa incidência de efeitos colaterais, destacando-se pelos efeitos analgésicos em diferentes síndromes dolorosas e neuropáticas, como adjuvantes ou em monoterapia.
\end{abstract}

Palavras-chave: Vitamina B12. Dor. Metilcobalamina. Neuropatia Periférica. 


\title{
POSSIBLE EVIDENCE OF VITAMIN B12 IN NEUROPATHIC PAIN Would it be a protective factor in Neurological Diseases?
}

\begin{abstract}
Introduction: The prevalence of patients with neuropathic pain in the general population can reach $10 \%$. When compared with other chronic pain syndromes, patients with neuropathic pain describe a worse state of mental and physical health, making their control challenging. Given the quantitative visible of diseases associated with neuropathic pain and the responses restricted to current management, the search for new therapies to be used as monotherapy or in combination with current treatments is revealed. One of these therapies is based on vitamin B12 supplementation, although no signs suggestive of deficiency are evident. Methodology: This is a descriptive exploratory study that uses the method of integrative review, a category that consists of exploring the knowledge already defined, including research with different techniques. In April 2020, a survey of articles indexed in the electronic databases PubMed, MedLine, LILACS and BIREM was carried out. Results and Discussion: For several years, vitamin B12 has been used in the form of monotherapy or combined with other drugs for the treatment of pain in various clinical conditions, such as inflammatory, neuropathic pain, neurodegenerative diseases, radiculopathy, etc. demonstrating positive evidence. Conclusion: Given the evidence, vitamin B12 presents itself as a potential candidate for the treatment of peripheral neuropathy with good safety and low incidence of side effects, standing out for the analgesic effects in different painful and neuropathic syndromes, as adjuvants or as monotherapy .
\end{abstract}

Keywords: B12 vitamin. Pain. Methylcobalamin. Peripheral neuropathy. 


\section{INTRODUÇÃO}

A Associação Internacional para Estudo da Dor (IASP) descreve a dor neuropática como sendo uma condição ocasionada por uma injúria ou doença do sistema nervoso somatossensorial. Tal caracterização se aplica a um vasto espectro heterogêneo de síndromes de dor que se manifestam com uma alta gama de sinais e sintomas, além de diversos mecanismos fisiopatológicos subjacentes ${ }^{1,2}$.

A dor neuropática possui origem central ou periférica. Patologias periféricas como consumo crônico de álcool, diabetes mellitus, lesões nervosas traumáticas, toxicidade de drogas e câncer, assim como condições centrais, como o acidente vascular encefálico, lesões medulares e esclerose múltipla fazem parte da etiologia da dor neuropática ${ }^{2,3}$. Vale ressaltar que nem todas as síndromes de dor possuem apenas uma origem somatossensorial fisiopatológica, por exemplo, a radiculopatia cervical caracteriza-se como uma síndrome de dor mista, na qual uma lesão nervosa causa uma síndrome de dor neuropática comum ${ }^{4}$.

A experiência sensitiva ocasionada pela dor neuropática é ampla. Muitas sensações são relatadas pelos pacientes, como dor espontânea, contínua, evocada e paroxística. Dentro do espectro da dor evocada temos a alodínia, que consiste na percepção dolorosa precipitada por estímulos não nocivos, além da hiperalgesia, que consiste em uma resposta exagerada a um estímulo nocivo. Os pacientes também relatam sensações como parestesia, que incluem percepções de pontadas, queimação, formigamento e choque elétrico. Tais sensações levam a importantes morbidades e efeitos deletérios na qualidade de vida ${ }^{5,6}$.

A prevalência de pacientes acometidos por dor neuropática na população em geral pode chegar a $10 \%^{7}$. Quando comparado com demais síndromes de dor crônica, os pacientes com dor neuropática descrevem pior estado de saúde mental e física, tornando-se desafiador seu controle. Utiliza-se de monoterapia farmacológica e tratamentos combinados para alivio da dor. Entretanto, tais esquemas terapêuticos estão relacionados a efeitos colaterais potencialmente graves, que podem não ser suportados pelos pacientes ${ }^{6}$.

Dado o visível quantitativo de doenças associadas à dor neuropática e as respostas restritas aos manejos atuais, revela-se a busca por novas terapêuticas a serem utilizadas como monoterapia ou em combinação com os tratamentos vigentes ${ }^{8}$. 
Uma dessas terapêuticas baseia-se na suplementação com vitamina B12, ainda que não seja evidenciado sinais sugestivos de deficiência. A B12, também nomeada como cobalamina, é uma vitamina com propriedades importantes para diversas funções biológicas. É uma substância neurotrófica com predileção para tecidos neuronais, sendo também reconhecida pela sua ação no que diz respeito a manutenção e regeneração dos nervos periféricos ${ }^{8}$.

Dentro de suas muitas funções, a B12 promove o processo de mielinização, levando à restauração funcional e regeneração de nervos através da regulação positiva da transcrição do gene. Além disso, a B12 inibe descargas ectópicas espontâneas dos neurônios sensoriais primários periféricos em estados de dor neuropática, o qual poderia explicar porque auxilia na atenuação de sintomas dolorosos ${ }^{9}$.

Com isso, o objetivo do presente estudo visa avaliar as principais evidências disponíveis na literatura vigente que destacam a vitamina B12 como alternativa à monoterapia ou terapia combinada para o tratamento da dor neuropática.

\section{METODOLOGIA}

Trata-se de estudo exploratório descritivo que emprega o método de revisão integrativa, categoria que consiste em explorar o conhecimento já definido, incluindo pesquisas com diferentes técnicas. Realizou-se em abril de 2021 o levantamento de artigos indexados nas bases de dados eletrônicas PubMed, MedLine, LILACS e BIREME.

Os termos de busca utilizados, segundo os Descritores em Ciências da Saúde (DeCS), foram, respectivamente em português, inglês e espanhol: "Vitamina B 12", "Vitamin B 12", "Vitamina B 12", "Traumatismos dos Nervos Periféricos", "Peripheral Nerve Injuries", "Traumatismos de los Nervios Periféricos". Para se incluir o maior número de artigos possível que abordassem o foco temático, a busca foi realizada selecionando os termos relevantes. Em todas as associações foi empregado o conectivo "and". Dessa maneira, utilizou-se as seguintes chaves de busca: "Vitamina B 12" and "Traumatismo dos Nervos Periféricos".

Priorizou-se por aplicar como filtro de pesquisa "textos completos", pois os autores entendem que é de suma importância o reconhecimento das obras disponíveis gratuitamente 
e, sobretudo, por considerarem que esses materiais são essenciais para promoverem acesso igualitário à informação.

Como critério de inclusão evidenciou-se a disponibilidade do artigo original completo indexado em português, inglês ou espanhol. Não contemplou os critérios de inclusão, aqueles que não tinham relação direta com o tema e outros tipos de produção, como dissertações, editoriais de revistas, teses, apresentação de pôsteres, anais de congresso.

Após levantamento preliminar, 24 artigos foram encontrados. Selecionados os estudos únicos, títulos e resumos foram analisados, selecionando aqueles que condiziam com o tema de interesse. Quando apenas a leitura dos títulos e resumo não foram suficientes para compreender o estudo, optou-se pela leitura integral do artigo.

Posteriormente a seleção cautelosa, foi iniciado a leitura na íntegra dos 24 artigos da amostra e organizado os resultados. Utilizou-se método de análise de conteúdo. Neste enfoque qualitativo, os materiais foram examinados e sistematizados, reconhecendo temas comuns, conformidades ou discrepâncias e tendências gerais.

\section{RESULTADOS E DISCUSSÃO}

A vitamina B12, também denominada de metilcobalamina, compõe uma família de compostos intitulados de maneira geral de cobalaminas. Configura-se como uma vitamina hidrossolúvel, sintetizada tão somente por microrganismos, encontrada em quase todos os tecidos animais e estocada primordialmente no fígado na forma de adenosilcobalamina ${ }^{10}$. Alimentos de origem animal, como ovos, leite e carne são fontes naturais de vitamina B12 na dieta humana. Além disso, existem diversas evidências dos efeitos antihiperalgésicos das vitaminas do complexo B, em especial a vitamina B12, em diferentes formas de dor neuropática, motivo pelo qual justifica o objetivo do presente trabalho ${ }^{11}$.

As vitaminas do complexo B são essenciais para a síntese de ácido nucleico e proteínas, bem como para a síntese da fosfatidilcolina. A fosfatidilcolina configura-se como um fosfolipídio da membrana celular, que se transforma em colina e posteriormente é empregada na síntese de acetilcolina, um importante neurotransmissor ${ }^{12}$.

Os prováveis mecanismos analgésicos e anti-neurálgicos das vitaminas B, principalmente a vitamina B12, apresentados em estudos experimentais incluem: 
estabilização da excitabilidade elétrica dos nervos impedindo descargas ectópicas; interação com os mediadores responsáveis pela dor, com posterior aumento da disponibilidade e eficácia da noradrenalina e da 5-hidroxitriptamina na via descendente inibitória da dor; reestruturação de fibras dos nervos deteriorados com recuperação das funções neuromusculares na hiperalgesia periférica e alodínia; ganhos significativos no transporte axonal, maximizando a velocidade de condução nervosa ${ }^{13}$.

Há vários anos, a vitamina B12 tem sido empregada sob a forma de monoterapia ou combinadas a demais fármacos para o tratamento de dor em diversas condições clínicas, como dor inflamatória e neuropática, demonstrando evidências positivas ${ }^{14}$.

A neuralgia pós-herpética e suas várias formas possui base na literatura vigente e diversos estudos apoiam a inclusão da B12 no tratamento convencional. Um estudo realizado por Xu et al. analisou o uso de $1000 \mu \mathrm{g}$ de metilcobalamina, via subcutânea pela manhã, num período de seis vezes por semana, por quatro semanas. O grupo controle recebeu metilcobalamina $500 \mu \mathrm{g}$, por via oral, três vezes ao dia ${ }^{9}$.

Os resultados revelaram que a intensidade da dor foi atenuada no grupo de intervenção, após sete dias, e assim permaneceu decaindo até a finalização do estudo, em 28 dias. Fora observado também, atenuação expressiva da dor no grupo que recebeu B12 oral, no entanto, significativamente menor quando comparado as infiltrações subcutâneas. Ainda no grupo B12 subcutâneo, fora observado redução em outras modalidades de dor, como alodínia e dor paroxística. Secundariamente, esses pacientes tiveram melhora em sua qualidade de vida e impacto positivo em suas atividades cotidianas. Não foram relatados efeitos colaterais advindos de quaisquer grupos $^{9}$.

Em uma coorte analisada por 28 dias, com 80 participantes portadores de neuralgia pós-herpética, divididos de maneira homogênea em quatro grupos fora administrado as seguintes soluções: um grupo recebeu 1000 mg de cobalamina por via subcutânea, outro recebeu 100 mg de tiamina por via subcutânea, outro recebeu infiltrações de lidocaína e o último recebeu uma associação de 100 mg de tiamina e B12 1000 g por via subcutânea. Ao final do estudo, observou-se expressiva redução da dor apenas nos grupos na qual a B12 estava inserida. Em concordância com os demais estudos, não foram relatados efeitos colaterais em quaisquer grupos ${ }^{15}$. 
Um estudo realizado em 98 pacientes portadores de neuralgia herpética oftálmica aguda, avaliou a infiltração local em contraste com a via intramuscular de metilcobalamina e lidocaína. Foram divididos em dois grupos. O grupo intervenção recebeu $1000 \mu \mathrm{g}$ de vitamina B12 associados a 20 mg de lidocaína, via subcutânea, uma vez ao dia. Por outro lado, o grupo controle recebeu 1000 ug de B12 mais 20 mg de lidocaína, por via intramuscular, uma vez ao dia. Ambos os grupos sofreram intervenções seis vezes por semana por um período de duas semanas $^{16}$.

Verificou-se que, apesar do grupo controle vivenciar melhorias significativas na severidade da dor, tal efeito permanecia por pouco tempo, atenuando paulatinamente após 14 dias. Contudo, fora observado que o grupo intervenção apresentou importante atenuação da intensidade da dor, sustentada por até um ano após a intervenção. O referido estudo, revela a superioridade da administração local de tais compostos em comparação com a via intramuscular para neuralgia herpética oftálmica aguda. Além disso, observou-se melhoras na qualidade de vida em ambos os grupos. Nenhum efeito colateral foi referido em todos os $\operatorname{grupos}^{16}$.

Ainda, segundo $\mathrm{Xu}$ et al., um estudo fora realizado em 204 pacientes. No referido estudo, foi avaliado o tratamento da manifestação troncular de neuralgia pós-herpética e não de neuralgia herpética oftálmica aguda. Ambos os grupos foram divididos e mediados de maneira similar. Observou-se que a intensidade da dor no grupo de intervenção fora superior ao grupo controle. Houve melhora na qualidade de vida de ambos os grupos, o que corrobora com os achados realizados no estudo anteriormente mencionado. Ademais, nenhum efeito adverso fora observado.

Levando em consideração esses resultados e os demais registros encontrados na literatura, existem evidências que apoiam a B12 como uma nova terapia na atenuação da dor, seja como terapia combinada ou monoterapia. Tal fato é sólido, e independe do local de manifestação da neuralgia pós-herpética ${ }^{17}$.

Em sua maioria, as bases de pesquisa descrevem a vitamina B12 como monoterapia ou terapia combinada para o tratamento da dor de pacientes com polineuropatia periférica de qualquer causa. Destacando as neuropatias diabéticas, neuropatias induzidas por quimioterapia e neuropatia a álcool ${ }^{16}$. 
Um estudo aberto randomizado, realizado por Mimenza Alvarado e Navarro, analisaram o gerenciamento da dor em uma população de pacientes portadores de neuropatia diabética de baixa e moderada intensidade. No referido estudo, as terapias confrontadas foram um regime de aumento de dosagem de Gabapentina, Tiamina e Cianocobalamina versus Pregabalina isolada administrada por via oral. Ao longo de 12 semanas de monitorização, ambos os grupos demonstraram importante atenuação da dor. Houve melhora significativa na qualidade do sono dos pacientes, com cada grupo referindo aumento no número de horas de sono ${ }^{18}$.

A monoterapia com vitamina B12 também foi avaliada em um estudo realizado por Talaei et al. Dois grupos foram estudados. O primeiro recebeu a dose de $2.000 \mu \mathrm{g}$ de vitamina B12, por via intra muscular, duas vezes por semana. Em contrapartida, $10 \mathrm{mg}$ de nortriptilina, por via oral, fora administrada no segundo grupo. Ao final, foi possível concluir que embora ambos os grupos tenham apresentado melhoras expressivas no que diz respeito a dor, o grupo que utilizou B12 apresentou atenuação significativamente maior em relação ao grupo nortriptilina ${ }^{19}$.

Evidências foram encontradas relatando a melhora dos sintomas dolorosos relacionados a neuropatia diabética urêmica com a inclusão de vitamina B12. No referido estudo de coorte prospectivo foram administrados $500 \mu \mathrm{g}$ de metilcobalamina por via intravenosa, três vezes por semana, por um período de seis meses em pacientes submetidos a hemodiálise regular com polineuropatia decorrente de uremia e diabetes. Observou-se melhoras significativas nos escores da dor neuropática ${ }^{20}$.

Ainda, fora avaliada a eficácia terapêutica da inclusão da vitamina B12 em 30 pacientes com lesões de raízes nervosas causadas por compressão, após excisão de hernia de disco. Todos os pacientes foram submetidos a eletroneuromiografia e avaliações no 10 ㅇ e 60 ㅇ dia após a cirurgia. Os autores do estudo observaram melhora clínica e eletrofisiológica estatisticamente importante com o uso terapêutico da vitamina B12. Ademais, concluíram que tal inclusão se aplica a radiculopatias de diferentes causas com expressivos ganhos na atenuação da dor ${ }^{24}$. 


\section{B12 como fator de proteção ou atenuação de doenças neurológicas?}

Uma coorte realizada com pacientes portadores de doenças neurodegenerativas visou observar se níveis baixos de B12 estariam associadas ao desenvolvimento de comprometimento cognitivo e progressão de tais doenças. O estudo fora realizado por meio de uma análise transversal dos níveis de vitamina B12 apurado no momento do diagnóstico em pacientes com Doença de Parkinson, Doença de Alzheimer, Atrofia Múltipla de Sistemas, Demência Frontotemporal, Demência com Corpos de Lewy, Paralisia Supranuclear Progressiva ou Comprometimento Cognitivo Leve. Apenas pacientes com mais de 3 anos de diagnóstico foram incluídos. Observou-se que os níveis de B12 eram significativamente mais baixos em pacientes com Doença de Parkinson, Doença de Alzheimer e Comprometimento Cognitivo Leve, embora uma certa redução dos níveis de B12 tenha sido observada nas demais condições $^{21}$.

Além das condições aqui já mencionadas, há registros na literatura que apoiam os promissores efeitos neuroprotetores da vitamina B12 em pacientes portadores de Esclerose Lateral Amiotrófica. Tais estudos atentam que a administração de metilcobalamina é responsável pela regeneração nervosa, podendo aumentar os potenciais de ação muscular desses pacientes, além disso, acredita-se que seus efeitos sobrepõem a ação analgésica, pressupõe que tal composto estaria atuando não só na melhora dos sintomas, mas no retardo da progressão da disfunção motora ${ }^{22}$.

Esses resultados apoiam e reafirmam a necessidade de suplementação de B12 nas doenças em questão. Como no estudo de um paciente apresentando história de 2 meses de distúrbios progressivos da marcha e mudanças comportamentais, com comprometimento da sensibilidade vibratória e sinais de lesão piramidal. Ao exame, fora evidenciado níveis baixos de vitamina B12 e a ressonância magnética revelou sinal hiperintenso nos tratos corticoespinais, um padrão similar ao observado na doença do neurônio motor. Foi implementado a terapia de reposição de B12, sendo então observado a melhora motora e cognitiva. Os autores concluíram que a encefalopatia relacionada aos níveis baixos de B12 era responsável por mimetizar aspectos de neuroimagem da doença do neurônio motor ${ }^{23}$. 


\section{CONCLUSÃO}

A vitamina B12 apresenta-se como um candidato potencial para o tratamento da neuropatia periférica com boa segurança e baixa incidência de efeitos colaterais, destacandose pelos efeitos analgésicos em diferentes síndromes dolorosas e neuropáticas, como adjuvantes ou em monoterapia. Embora mais pesquisas sejam necessárias, as evidências vigentes apontam a vitamina B12 como uma alternativa segura e de baixo custo, configurando-se como uma excelente opção para tratamentos álgicos.

\section{REFERÊNCIAS}

The IASP Subcommittee on Taxonomy Pain terms a list with definitions and notes on usage. Recommended by the IASP subcommittee on taxonomy. 1979, 6:249.

Bouhassira D. Neuropathic pain: Definition, assessment and epidemiology. Rev. Neurol. 2018 175:16-25. doi: 10.1016/j.neurol.2018.09.016.

Brozou V., Vadalouca A., Zis P. Pain in platin-induced neuropathies: A systematic review and meta-analysis. Pain Ther. 2018 7:105-119.

Murphy D.R., Hurwitz E.L., Gerrard J.K., Clary R. Pain patterns and descriptions in patients with radicular pain: Does the pain necessarily follow a specific dermatome? Chiropr. Osteopat. 2009 17:9. doi: 10.1186/1746-1340-17-9.

Girach A., Julian T.H., Varrassi G., Paladini A., Vadalouka A., Zis P. Quality of life in painful peripheral neuropathies: A systematic review. Pain Res. Manag. 2019 23:1-9. doi: 10.1155/2019/2091960.

Freynhagen R., Bennett M.I. Diagnosis and management of neuropathic pain. BMJ. 2009 339:b3002. doi: 10.1136/bmj.b3002.

van Hecke O., Austin S.K., Khan R.A., Smith B.H., Torrance N. Neuropathic pain in the general population: A systematic review of epidemiological studies. Pain. 2014 155:654662. doi: 10.1016/j.pain.2013.11.013.

Huang Z.-F., Lin B.-Q., Torsha T.T., Dilshad S., Yang D.-S., Xiao J. Effect of mannitol plus vitamins $B$ in the management of patients with piriformis syndrome. J. Back Musculoskelet. Rehabil. 2019 32:329-337. doi: 10.3233/BMR-170983.

Xu G., Lv Z.-W., Feng Y., Tang W.-Z., Xu G.X. A single-center randomized controlled trial of local methylcobalamin injection for subacute herpetic neuralgia. Pain Med. 2013 14:884894. doi: 10.1111/pme.12081

Henry, J. B. Diagnósticos clínicos e tratamento por métodos laboratoriais. 2. ed. São Paulo: Manole Ltda, 1999. Cap. 26, p. 621-5. 
Fairbanks, v. F.; klee, g. G. Aspectos bioquímicos da hematologia. In: burtis, C. A.; ashwood, E. R. Tietz: fundamentos de química clínica. 4. ed. Rio de Janeiro: Guanabarra Koogan S. A., 1998. Cap. 36, p. 699-703.

Granados-Soto V, Sánchez-Ramírez G, la Torre MR, Caram-Salas NL, Medina-Santillán R, Reyes-García G. Effect of diclofenac on the antiallodynic activity of vitamin B12 in a neuropathic pain model in the rat. Proc West Pharmacol Soc. 2004;47:92-4.

Zhang M, Han W, Hu S, Xu H. Methylcobalamin: a potential vitamin of pain killer. Neural Plast. 2013;2013;424651

Pérez-Flores E, Medina-Santillán R, Reyes-García G, Mateos-García E. Combination of diclofenac plus $B$ vitamins in acute pain after tonsillectomy: a pilot study. Proc West Pharmacol Soc. 2003;46:88-90.

Xu G., Lv Z.W., Xu G., Tang W.Z. Thiamine, cobalamin, locally injected alone or combination for herpetic itching: A single-center randomized Controlled Trial. Clin. J. Pain. 2014 30:269278. doi: 10.1097/AJP.0b013e3182a0e085.

Xǔ G., Xu S., Cheng C., Xú G., Tang W.-Z., Xu J. Local administration of methylcobalamin and lidocaine for acute ophthalmic herpetic neuralgia: A single-center randomized controlled trial. Pain Pract. 2016 16:869-881. doi: 10.1111/papr.12328.

Xǔ G., Xu S., Tang W.-Z., Xú G., Cheng C., Xu J. Local Injection of Methylcobalamin Combined with Lidocaine for Acute Herpetic Neuralgia. Pain Med. 2015 17:572-581. doi: 10.1093/pm/pnv005.

Mimenza Alvarado A., Aguilar Navarro S. Clinical trial assessing the efficacy of gabapentin plus $b$ complex (b1/b12) versus pregabalin for treating painful diabetic neuropathy. J. Diabetes Res. 2016 :4078695. doi: 10.1155/2016/4078695.

Talaei A., Siavash M., Majidi H., Chehrei A. Vitamin B 12 may be more effective than nortriptyline in improving painful diabetic neuropathy. Int. J. Food Sci. Nutr. 2009 60(Suppl. 5):71-76. doi: 10.1080/09637480802406153.

Kuwabara S., Nakazawa R., Azuma N., Suzuki M., Miyajima K., Fukutake T., Hattori T. Intravenous methylcobalamin treatment for uremic and diabetic neuropathy in chronic hemodialysis patients. Intern. Med. 1999 38:472-475. doi: 10.2169/internalmedicine.38.472.

Luthra NS, Marcus AH, Hills NK, Christine CW. Vitamin B12 measurements across neurodegenerative disorders. J Clin Mov Disord. 2020; 7: 3. doi: 10.1186/s40734-02000085-8

Ikeda K, Iwasaki Y, Kaji R. Neuroprotective effect of ultra-high dose methylcobalamin in wobbler mouse model of amyotrophic lateral sclerosis. J Neurol Sci. 2015, 15;354(1-2):704. doi: 10.1016/j.jns.2015.04.052. 
Pinto WBVR, de Souza PVS, Rogério RM, Pedroso JL, Barsottini OGP. Vitamin B12 deficiency mimicking neuroimaging features of motor neuron disease. Arq. Neuro-Psiquiatr. 2014, 72 (1).

E Alfonsi , GF Berzero , C Zandrini , G Felicetti. The role of drug therapy in the treatment of nerve root lesions caused by compression. Clinical and electrophysiological study of 30 patients operated on for the removal of herniated disks. Minerva Med. 1984, 14;75(2829):1727-32. 